\title{
On The Design Of Output Feedback Controllers For Continuous-Time LTI Systems Over Fading Channels
}

\author{
L. $\mathrm{Su}^{\mathrm{a}^{*}}, \mathrm{G} \cdot \mathrm{Chesi}{ }^{\mathrm{a}}$ \\ a Department of Electrical and Electronic Engineering, The University of Hong Kong, Pokfulam Road, Hong Kong \\ *Corresponding Author: 1lsu@eee.hku.hk
}

\begin{abstract}
This paper considers continuous-time linear timeinvariant (LTI) systems over fading channels, and addresses the design of output feedback controllers that stabilize the closed-loop system in the mean square sense. It is shown that a sufficient and necessary condition for the existence of such controllers can be obtained by solving a convex optimization problem in the form of a semidefinite program (SDP). This condition is obtained by introducing a modified Hurwitz stability criterion and by exploiting polynomials that can be written as sums of squares of polynomials.
\end{abstract}

Keywords: Fading channels, Mean square stability, Output feedback control, SDP.

\section{Introduction}

Networked control systems have progressively become one of the hottest topics in recent years ${ }^{(1,2,3)}$. In this area, stability and stabilization have been studied in the presence of information constraints, e.g., finite communication bandwidth $^{(4)}$, quantizers with limited information ${ }^{(5)}$, time delays ${ }^{(6)(7)}$, unreliable networks with packet losses ${ }^{(8)}$, fading channels $^{(9)}$, channel with resource allocation ${ }^{(10)}$, signal-tonoise constraints ${ }^{(11)}$.

Recently, the work ${ }^{(9)}$ has investigated networked control systems with fading channels. Here the connections between plant and controller are affected by multiplicative white noise processes. Sufficient and necessary conditions for stability in the mean square sense are provided. Also, sufficient and necessary conditions for the existence of stabilizing state feedback controllers are provided.

This paper aims at generalizing the results provided in the work $^{(9)}$ by considering the design of output feedback controllers for continuous-time LTI systems over fading channels. Specifically, a continuous-time LTI plant is controlled in closed-loop by an output feedback controller, which is connected through fading channels modeled as multiplicative white noise processes. The problem consists of establishing the existence of controllers in a desired semialgebraic set such that the closed-loop system is stable in the mean square sense. First, an equivalent reformulation of mean square stability is given as asymtotical stability of a suitable matrix. Second, an equivalent reformulation of Hurwitz matrx is derived based on a modified Hurwitz stability criterion. Third, a sufficient and necessary condition for the existence of stabilizing output feedback controllers is obtained based on the solution of a convex optimization problem in the form of an SDP by exploiting polynomials that can be written as sums of squares of polynomials.

The paper is organized as follows. Section 2 introduces some preliminaries. Section 3 describes the proposed results. Section 4 presents the illustrative example. Section 5 reports the conclusions.

\section{Preliminaries}

The notation used in the paper is as follows. The sets of real numbers and complex numbers are denoted by $\mathbb{R}$ and $\mathbb{C}$. The notation $\mathcal{R}(\lambda)$ denotes the real part of a complex number $\lambda$. The inverse and the transpose of a matrix $A$ are denoted by $A^{-1}$ and $A^{\prime}$. The notation $\operatorname{det}(A)$ denotes the determinant of a matrix $A$. The spectrum of a matrix $A$, i.e., the set of eigenvalues of $A$, is denoted by $\operatorname{spec}(A)$. The vector obtained by stacking all the columns of a matrix $A$ into one column vector is denoted by $\operatorname{vec}(A)$. The notation $I$ denotes the identity matrix with the size 


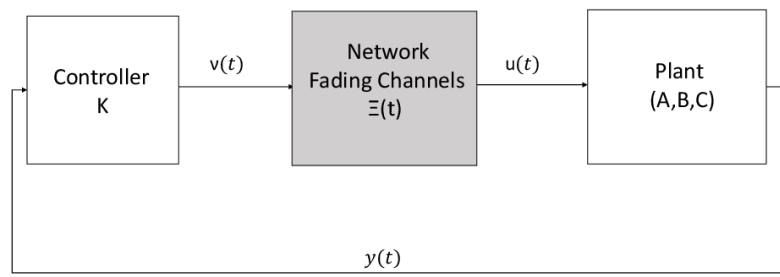

Fig. 1. Output Feedback Control over Fading Channels.

specified in the context. For scalars $a_{1}, \ldots, a_{n}$, the notation $\operatorname{diag}\left(a_{1}, \ldots, a_{n}\right)$ denotes the diagonal matrix with its $(i, i)$-th entry equal to $a_{i}, i=1, \ldots, n$. The degree of a polynomial $p(\cdot)$ is denoted by $\operatorname{deg}(p(\cdot))$. The symbol $\otimes$ denotes the Kronecker product. The operator $\mathcal{E}(\cdot)$ denotes the mathematical expectation.

\subsection{Problem Formulation}

Let us consider the situation depicted in Figure 1 where a plant is controlled in closed-loop by an output feedback controller over fading channels.

The plant is described by

$\left\{\begin{array}{llr}\dot{x}(t) & = & A x(t)+B u(t) \\ y(t) & = & C x(t)\end{array}\right.$

where $x(t) \in \mathbb{R}^{n}$ is the plant state, $u(t) \in \mathbb{R}^{m}$ is the plant

input, and $y(t) \in \mathbb{R}^{p}$ is the plant output.

The controller is initially supposed static for clarity of presentation $^{1}$, and is described by

$v(t)=K y(t)$

where $v \in \mathbb{R}^{m}$ is the controller output and $K \in \mathbb{R}^{m \times p}$ has to be determined in the semi-algebraic set

$\mathcal{K}=\left\{K \in \mathbb{R}^{m \times p}: a_{i}(K) \geq 0, i=1, \ldots, n_{a}\right\}$

\footnotetext{
${ }^{1}$ As it will be explained in Remark 2, the proposed methodology can be used also to design dynamic output feedback controllers.
}

where $a_{i}(K)$ are polynomials.

The fading channels are modeled in memoryless multiplicative form as

$u(t)=\Xi(t) v(t)$

where $\Xi(t) \in \mathbb{R}^{m \times m}$ represents the channel fading and has the diagonal structure

$\Xi(t)=\operatorname{diag}\left\{\xi_{1}(t), \xi_{2}(t), \ldots, \xi_{m}(t)\right\}$

where $\xi_{1}(t), \ldots, \xi_{m}(t)$ are assumed to be scalar-valued white noise processes with

$\mu_{i}=\mathcal{E}\left(\xi_{i}(t)\right) \neq 0 \forall i=1, \ldots, m$.

Let us define

$\Pi=\operatorname{diag}\left(\mu_{1}, \ldots, \mu_{m}\right)$.

and

$\Sigma=\left[\sigma_{i j}\right]_{i, j=1, \ldots, m}$

where

$\sigma_{i j}=\mathcal{E}\left(\left(\xi_{i}(t)-\mu_{i}\right)\left(\xi_{j}(t)-\mu_{j}\right) \forall i, j=1, \ldots, m\right.$

Denote $\sigma_{i}=\sqrt{\sigma_{i i}}, i=1, \ldots, m$. It is assumed that the fading channel $\Xi(t)$ and the initial state $x(0)$ are uncorrelated.

The closed-loop system obtained by connecting the output of the controller (2) to the input of the unstable plant (1) over the fading channels (4) can be described by

$$
\dot{x}(t)=A_{c l}(K, t) x(t)
$$

where

$A_{c l}(K, t)=A+B \Xi(t) K C$.

Let us further define

$$
X(t)=\mathcal{E}\left(x(t) x(t)^{\prime}\right) .
$$

Definition $1^{(12)}$ The closed-loop system (10) is said to be stable in the mean square sense if $X(t)$ is well-defined for all $t \geq 0$ and

$\lim _{t \rightarrow \infty} X(t)=0 \forall x(0) \in \mathbb{R}^{n}$. 
The basic problem addressed in this paper is as follows.

Problem 1 Design a static output controller $K$ in the set $\mathcal{K}$ such that the closed-loop system (10) is stable in the mean square sense.

\subsection{Modified Routh-Hurwitz Table}

The Routh-Hurwitz stability criterion provides a necessary and sufficient condition for establishing whether the roots of a univariate polynomial with real coefficients have negative real parts ${ }^{(13)}$. The entries of this table are rational functions of the coefficients of the polynomial. Hereafter, we introduce a modified Routh-Hurwitz table where the entries are polynomial functions of these coefficients instead of rational functions ${ }^{(14)}$.

Let us denote the characteristic polynomial of $A$ as

$$
v(s)=\operatorname{det}(s I-A)=s^{n}+a_{n-1} s^{n-1}+\ldots+a_{0}
$$

where $s \in \mathbb{C}$ and $a_{n-1}, \ldots, a_{0} \in \mathbb{R}$ are the coefficients of $v(s)$. By multiplying each entry by their denominator, one obtains the modified Routh-Hurwitz table defined as

$$
\begin{array}{cccc}
1 & a_{n-2} & a_{n-4} & \cdots \\
a_{n-1} & a_{n-3} & a_{n-5} & \cdots \\
a_{31} & a_{32} & a_{33} & \cdots \\
\vdots & \vdots & \vdots & \ddots
\end{array}
$$

where the number of rows is $n+1$ and the $i j$-th entry is

$a_{i j}=a_{i-1,1} a_{i-2, j+1}-a_{i-1, j+1} a_{i-2,1}$

$i=3, \ldots, n+1, j=1,2 \ldots$

It can be easily shown that all the roots of $v(s)$ have negative real parts if and only if the first column of the modified Routh-Hurwitz table contains positive entries only.

\subsection{SOS Polynomials}

Here we briefly introduce the class of sums of squares
(SOS) polynomials ${ }^{(15)}$

A polynomial $p(s): \mathbb{R}^{r} \rightarrow \mathbb{R}$ is said to be SOS if and only if there exist polynomials $p_{1}(s), \ldots, p_{k}(s): \mathbb{R}^{r} \rightarrow \mathbb{R}$ such that

$$
p(s)=\sum_{i=1}^{k} p_{i}(s)^{2}
$$

SOS polynomials are nonnegative for any value of the variable being the sum of squares of real numbers. Moreover, one can establish whether a polynomial is SOS via an LMI feasibility test.

Indeed, let $d$ be a nonnegative integer such that $2 d \geq \operatorname{deg}(p)$. Then, $p(s)$ can be written according to the Gram matrix method, or square matrix representation (SMR), as

$p(s)=b(s)^{\prime}(G+L(\alpha)) b(s)$

where $b: \mathbb{R}^{r} \rightarrow \mathbb{R}^{\sigma(r, d)}$ is a vector containing all the monomials of degree less than or equal to $d$ in $S$ and

$\sigma(r, d)=\frac{(r+d) !}{r ! d !}$

$G \in \mathbb{R}^{\sigma(r, d) \times \sigma(r, d)}$ is a symmetric matrix satisfying

$p(s)=b(s)^{\prime} G b(s)$,

$L: \mathbb{R}^{\omega(r, 2 d)} \rightarrow \mathbb{R}^{\sigma(r, d) \times \sigma(r, d)}$ is a linear parametrization of the linear set

$\mathcal{L}=\left\{\tilde{L}=\tilde{L}{ }^{\prime}: b(s)^{\prime} \tilde{L} b(s)=0\right\}$,

and $\alpha \in \mathbb{R}^{\omega(r, 2 d)}$ is a free vector with

$\omega(r, 2 d)=\frac{1}{2}(\sigma(r, d)(\sigma(r, d)+1))-\sigma(r, 2 d)$.

It follows that $p(s)$ is SOS if and only if there exists $\alpha$ satisfying the LMI

$G+L(\alpha) \geq 0$

\section{Proposed Approach}

\subsection{Stability Analysis}

In this subsection, we consider the stability analysis of the closed-loop systems (10) over fading channels.

Let us consider $X(t)$ in (12), and let us define the 
vector

$v_{l}(X(t))=\operatorname{vec}(X(t))$.

Also, let us denote with $v_{s}(X(t))$ the same vector where the entries of $X(t)$ that lie under its diagonal have been removed. It follows that

$v_{l}=G v_{s}$

where $G \in \mathbb{R}^{n^{2} \times \frac{1}{2} n(n+1)}$. Let us observe that $G$ is a full column rank matrix. Let $B_{i}$ denote the $i$-th column of $B$, and let $(K C)_{i}$ denote the $i$-th row of $K C$.

Theorem 1 The closed-loop system (10) is stable in the mean square sense if and only if $\mathcal{R}(\lambda)<0, \forall \lambda \in \operatorname{spec}(\Phi)$, where

$\Phi(K)=\left(G^{\prime} G\right)^{-1} G^{\prime} \Psi(K) G$

where

$$
\begin{aligned}
\Psi=I \otimes & (A+B \Pi K C)+(A+B \Pi K C) \otimes I \\
& +\sum_{i=1}^{m} \sum_{j=1}^{m} \sigma_{i j}\left(B_{i}(K C)_{i}\right) \otimes\left(B_{j}(K C)_{j}\right) .
\end{aligned}
$$

Proof. The closed-loop system can be rewritten into the It $\hat{o}$ form as follows:

$d x(t)=(A+B \Pi K C) x(t) d t+\sum_{i=1}^{m} \sigma_{i} B_{i}(K C)_{i} x(t) d \omega_{i}(t)$

where $\omega_{i}(t), i=1, \ldots, m$ are possibly correlated standard scalar Wiener processes with $d \omega_{i} \cdot d \omega_{j}=\frac{\sigma_{i j}}{\sigma_{i} \sigma_{j}} d t, i, j=1, \ldots, m$. According to the It $\hat{o}$ 's formula, one has

$$
\dot{X}(t)=(A+B \Pi K C) X(t)+X(t)(A+B \Pi K C)^{\prime}+
$$

$\sum_{i=1}^{m} \sum_{j=1}^{m} \sigma_{i j}\left(B_{i}(K C)_{i}\right) X(t)(K C)_{j}^{\prime} B_{j}^{\prime}$

where $X(t)$ is defined in (12). Hence, it can be observed that

$\operatorname{vec}(\dot{X}(t))=\Psi \operatorname{vec}(X(t))$ where $\Psi$ is defined in (27), which follows that $\lim _{t \rightarrow \infty} X(t)=0 \quad$ if and only if $\mathcal{R}(\lambda)<0, \forall \lambda \in \operatorname{spec}(\Psi)$.

Since $X(t)$ is a symmetric matrix, one can remove the replicated entries using (25). Then, it can be obtained that

$G v_{s}(\dot{X}(t))=\Psi G v_{s}(X(t))$,

where $G$ is a full column rank constant matrix. After premultiplying the above equation by $\left(G^{\prime} G\right)^{-1} G^{\prime}$, one has

$v_{s}(\dot{X}(t))=\left(G^{\prime} G\right)^{-1} G^{\prime} \Psi G v_{s}(X(t))$.

where $\Phi$ is defined as (26)-(27).

Observe that $\lim _{t \rightarrow \infty} X(t)=0 \quad$ is equivalent to $\lim _{t \rightarrow \infty} v_{s}(X(t))=0$, which follows that the closed-loop system (10) is stable in the mean square sense if and only if $\mathcal{R}(\lambda)<0, \forall \lambda \in \operatorname{spec}(\Phi)$ where $\Phi$ is defined as (26)-

Let us observe that $\Phi(K)$ can be considered as a matrix alternative to $\Psi(K)$ for describing the closed-loop system.

\subsection{Controller Synthesis}

Let us start by defining the new variable $k \in \mathbb{R}^{n_{k}}$ as

$k=\operatorname{vec}(K)$,

and let us define

$n_{\Phi}=\frac{1}{2} n(n+1)$

which is the size of the square matrix $\Phi(K)$. Let us denote the characteristic polynomial of $\Phi(K)$ as

$$
\begin{aligned}
v(s, k)= & \operatorname{det}(s I-\Phi(K)) \\
& =s^{n_{\Phi}}+\sum_{j=0}^{n_{\Phi}{ }^{-1}} c_{j}(k) s^{j}
\end{aligned}
$$

where $s \in \mathbb{C}$ and $c_{0}(k), \ldots, c_{n_{\Phi^{-1}}}(k) \in \mathbb{R} \quad$ are the coefficients of $v(s, k)$. 
Next, let us exploit the modified Routh-Hurwitz table to derive an equivalent condition of Theorem 1 . Let $T$ be the modified Routh-Hurwitz table of the characteristic polynomial (30). As defined in 2.2, one can observe that all the entries of the modified table are polynomials with respect to the variable $k$. Denote the number of entries in the first column of $T$ with $n_{f}$ and let $f_{i}(k)=T_{i 1}, i=1,2, \ldots, n_{f}$, which represents the first column of the table $T$. To this end, let us further define the set

$\hat{\mathcal{K}}=\left\{K \in \mathbb{R}^{m \times p}: f_{i}(k)-\varepsilon \geq 0, i=1, \ldots, n_{f}-1\right\}$

where $\varepsilon>0$ is introduced for considering positive values only of $f_{i}(k), i=1,2, \ldots, n_{f}-1$. Observe that there is no need to go on with controller synthesis if any $f_{i}(k)$ is a nonpositive constant number since in that case the plant is not stabilizable.

Theorem 2 Let us define

$$
r=\sup _{K \in \mathcal{K} \cap \hat{\mathcal{K}}} f_{n_{f}}(k)
$$

where $f_{n_{f}}(k)$ is the last entry in the first column of the table $T$. There exists a controller $K^{*} \in \mathcal{K}$ such that the closed-loop system (10) is stable in the mean square sense if and only if

$$
r>0
$$

for some $\varepsilon>0$.

Proof. " $\Rightarrow "$ Suppose there exists a controller $K^{*} \in \mathcal{K}$ such that the closed-loop system (10) is stable. This implies that there exists $\varepsilon>0$ such that $K^{*} \in \hat{\mathcal{K}}$. Moreover, $f_{n_{f}}\left(k^{*}\right)>0$, and hence $r>0$.

$" \Leftarrow "$ Suppose $r>0$. This implies that there exist $k^{*} \in \mathcal{K} \cap \hat{\mathcal{K}}$ such that $f_{n_{f}}\left(k^{*}\right)>0$, which implies that the first column of the table $T$ contains only positive entries with $k=k^{*}$. Thus, there exists a controller $K^{*} \in \mathcal{K}$ such that the closed-loop system (10) is stable in the mean square sense.

Let us observe that, if $r>0$ for some $\varepsilon=\hat{\varepsilon}>0$, then $r>0$ for all $\varepsilon \in(0, \hat{\varepsilon}]$. This means that, in practice, $\varepsilon$ can be simply chosen a priori as the smallest positive number allowed by the available computing platform.

Let us introduce the following assumption.

Assumption 1 The semi-algebraic set $\mathcal{K}$ is compact, and the polynomials

$$
f_{i}(k), i=1, \ldots, n_{f}-1
$$

and $a_{j}(k), j=1, \ldots, n_{a}$ have even degrees and their highest degree forms have no common zeros except 0 .

Let us observe that Assumption 1 does not introduce strong restrictions. Indeed, it is reasonable to search for a controller in a compact set. Moreover, if the polynomials $f_{i}(k)$ and $a_{j}(k)$ have not even degrees, one can enforce this property without changing the problem by simply multiplying these polynomials by a linear function that is positive over $\mathcal{K}$. Lastly, the property that the highest degree forms of $f_{i}(k)$ and $a_{j}(k)$ have no common zeros except 0 is automatically satisfied for typical semi-algebraic set $\mathcal{K}$ such as hyper-ellipsoids, hyper-rectangles, etc.

For $\theta \in \mathbb{R}$, let us define the polynomial

$$
\begin{gathered}
g(k)=\theta-f_{n_{f}}(k)-\sum_{i=1}^{n_{f}^{-1}}\left(f_{i}(k)-\varepsilon\right) \gamma_{i}(k) \\
-\sum_{j=1}^{n_{a}} a_{j}(k) \beta_{j}(k) .
\end{gathered}
$$

Theorem 3 Suppose that Assumption 1 holds. Then, the condition (33) holds if and only if

$\theta^{*}>0$

where

$\theta^{*}=\inf _{\theta, \gamma_{i}, \beta_{j}} \theta$

$$
\text { s.t. }\left\{\begin{array}{rlr}
g(k) & \text { is } & \mathrm{SOS} \\
\gamma_{i}(k) \text { is } & \mathrm{SOS} \\
\beta_{j}(k) \text { is } & \mathrm{SOS} \\
\forall i & = & 1, \ldots, n_{f}-1 \\
\forall j & = & 1, \ldots, n_{a} .
\end{array}\right.
$$

Proof. $\quad \Rightarrow$ " Let us assume $r>0$. Based on the definition of $r$ in (32), one has $\theta^{\#}=r$ 
where $\theta^{\#}$ is defined as

$$
\theta^{\#}=\inf _{\theta} \theta \text { s.t. } \theta-f_{n_{f}}(k)>0 \forall K \in \mathcal{K} \cap \hat{\mathcal{K}} \text {. }
$$

Since Assumption 1 holds, it follows from the work ${ }^{(16)}$ that $\theta-f_{n_{f}}(k)>0$ for all $K \in \mathcal{K} \cap \hat{\mathcal{K}}$ if and only if

$$
\left\{\begin{array}{l}
\exists s_{0,} \gamma_{1}, \ldots, \gamma_{n_{f}-1}, \beta_{1}, \ldots, \beta_{n_{a}} \in \operatorname{SOS} \\
\theta-f_{n_{f}}(k)=s_{0}+\sum_{i=1}^{n_{f}-1}\left(f_{i}(k)-\varepsilon\right) \gamma_{i}(k) \\
\quad+\sum_{j=1}^{n_{a}} a_{j}(k) \beta_{j}(k) .
\end{array}\right.
$$

Hence, the condition (35)-(36) holds with $\theta^{*}=\theta^{\#}$.

$" \Leftarrow$ " Let us assume the (35)-(36) holds, and suppose by contradiction that $r \leq 0$. It follows that any $\theta>r$ will satisfy that $\theta-f_{n_{f}}(k)>0$ for all $K \in \mathcal{K} \cap \hat{\mathcal{K}}$. Thus, the optimal solution of $\theta^{*}$ in (36) should be less than or equal to 0 , contradicting (35), which completes the proof.

Theorem 3 states that one can establish whether $r>0$ by solving the optimization problem (36), which is an SDP. In particular, this theorem provides a sufficient condition for any chosen degrees of the polynomials $\gamma_{i}(k)$ and $\beta_{j}(k)$. Moreover, this condition is also necessary when these degrees are large enough.

The following theorem explains how one can use the SDP (36) to determine a controller that solves Problem 1.

Theorem 4 Suppose that Assumption 1 holds. Let $\hat{\theta}$ be the solution of the SDP (36) with specified degrees of the polynomials $\gamma_{i}(k)$ and $\beta_{j}(k)$. Then,

$\hat{\theta}=r$

if and only if there exists $K$ such that

$$
\left\{\begin{array}{ccc}
\hat{\theta}-f_{n_{f}}(k) & = & 0 \\
g^{*}(k) & = & 0 \\
K & \in \mathcal{K} \cap \hat{\mathcal{K}} .
\end{array}\right.
$$

Proof. " $\Rightarrow$ "Suppose (37) holds. Then, the first and the third conditions in (38) hold with the maximizer $K^{*}$ of (32). It follows that

$$
g^{*}(k)=\hat{\theta}-f_{n_{f}}(k)-\sum_{i=1}^{n_{f}^{-1}}\left(f_{i}(k)-\varepsilon\right) \gamma_{i}^{*}(k)
$$

$$
-\sum_{j=1}^{n_{a}} a_{j}(k) \beta_{j}^{*}(k)
$$

where $\gamma_{i}^{*}(k), \beta_{j}^{*}(k)$ are the optimal solution in (36). Let $k^{*}$ be $k$ defined for $K$ replaced by $K^{*}$, then one has $g^{*}\left(k^{*}\right)=-\sum_{i=1}^{n_{f}-1}\left(f_{i}\left(k^{*}\right)-\varepsilon\right) \gamma_{i}^{*}\left(k^{*}\right)-\sum_{j=1}^{n_{a}} a_{j}(k) \beta_{j}^{*}\left(k^{*}\right)$.

Let us observe that $K \in \mathcal{K} \cap \hat{\mathcal{K}}$ guarantees that $\left\{\begin{array}{cc}f_{i}\left(k^{*}\right)-\varepsilon \geq 0 & i=1, \ldots, n_{f}-1 \\ a_{j}(k) \geq 0 & j=1, \ldots, n_{a}\end{array}\right.$ and that (36) ensures that $g^{*}(k), \gamma_{i}^{*}(k)$ and $\beta_{j}^{*}(k)$ are SOS. Hence,

$0 \leq g^{*}\left(k^{*}\right) \leq 0$, making the second line of (38) hold. " $\Leftarrow$ "Suppose that (38) holds for some $K$. As implied in Theorem 3, $\hat{\theta}$ is an upper bound of $r$. Hence, (38) states that the upper bound $\hat{\theta}$ can be achieved by some $K \in \mathcal{K} \cap \hat{\mathcal{K}}$

Searching for a controller $K$ such that the condition (38) is satisfied can be done by, firstly, computing the set $\Omega=\left\{k \in \mathbb{R}^{n_{k}}: g^{*}(k)=0\right\}$.

Since $g^{*}(k)$ is a SOS polynomial, the set $\Omega$ can be found through computing vector of monomials in $k$ belonging to the null space of a positive semidefinite Gram matrix of $g^{*}(k) .^{(17)}$ After $\Omega$ has been found, one should check whether any $K$ in the set $\Omega$ satisfies the other two constraints of (38).

Remark 1 The procedure for determining a controller that solves Problem 1 can be summarized as follows. First, we build the modified Routh-Hurwitz table as described in 2.2 
for (26). Then, we build and solve the SDP (36). Lastly, we check feasibility of (38). In the case (38) is infeasible, one can increase the degrees of the polynomials $\gamma_{i}(k)$ and $\beta_{j}(k)$ and repeat.

Remark 2 Let us observe that the proposed methodology can be used not only to design static output feedback controllers as considered in (2), but also dynamic output feedback controllers. Indeed, this can be achieved as follows. First, one should replace (2) with

$\left\{\begin{array}{c}\dot{x}_{c}=A_{c} x_{c}(t)+B_{c} y(t) \\ v(t)=C_{c} x(t)+D_{c} y(t)\end{array}\right.$

where $\quad x_{c}(t) \in \mathbb{R}^{n_{c}} \quad$ is the controller state, and $A_{c} \in \mathbb{R}^{n_{c} \times n_{c}}, \quad B_{c} \in \mathbb{R}^{n_{c} \times p}, \quad C_{c} \in \mathbb{R}^{m \times n_{c}} \quad$ and $\quad D_{c} \in \mathbb{R}^{m \times p}$ are matrices to determine. Second, one should replace (28) with

$k=\left(\begin{array}{c}\operatorname{vec}\left(A_{c}\right) \\ \operatorname{vec}\left(B_{c}\right) \\ \operatorname{vec}\left(C_{c}\right) \\ \operatorname{vec}\left(D_{c}\right)\end{array}\right)$.

Lastly, one should replace (27) with

$\Psi=I \otimes(\bar{A}+\bar{B} \Pi \bar{C})+(\bar{A}+\bar{B} \Pi \bar{C}) \otimes I+$

$$
\sum_{i=1}^{m} \sum_{j=1}^{m} \sigma_{i j}\left(\bar{B}_{i} \bar{C}_{i}\right) \otimes\left(\bar{B}_{j} \bar{C}_{j}\right)
$$

where

$$
\left\{\begin{aligned}
\bar{A}(k) & =\left(\begin{array}{cc}
A & 0 \\
B_{c} C & A_{c}
\end{array}\right) \\
\bar{B} & =\left(\begin{array}{l}
B \\
0
\end{array}\right) \\
\bar{C}(k) & =\left(\begin{array}{ll}
D_{c} C & C_{c}
\end{array}\right) .
\end{aligned}\right.
$$

\section{Examples}

In this section, we present a numerical example to illustrate the proposed results. The computations are done by Matlab with the toolboxes SeDuMi ${ }^{(18)}$ and SOSTOOLS ${ }^{(19)}$.
The set $\hat{\mathcal{K}}$ is defined with $\varepsilon=10^{-3}$.

Let us consider the plant (1) with

$$
A=\left(\begin{array}{cc}
0.5 & -1 \\
1 & 0.5
\end{array}\right), B=\left(\begin{array}{l}
1 \\
1
\end{array}\right), C=\left(\begin{array}{ll}
1 & 1
\end{array}\right)
$$

and the fading channel (7)-(8) with

$\left\{\begin{aligned} \Pi= & 0.9 \\ \Sigma & =0.1\end{aligned}\right.$

The problem consists of determining a controller (2) over the set $\mathcal{K}=[-2,2]$ such that the closed-loop system (10) is

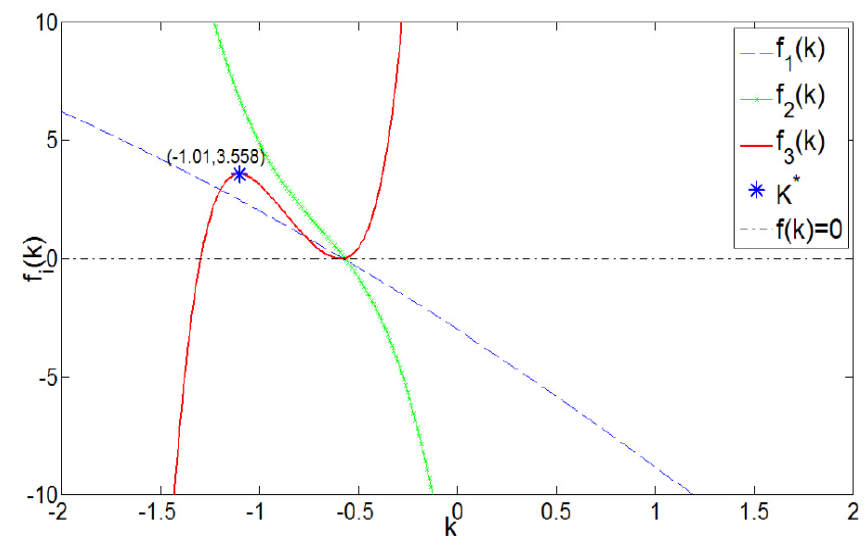

Figure 2: $f_{1}(k), f_{2}(k), f_{3}(k)$ versus $k$

stable in the mean square sense.

First of all, let us observe that the autonomous system (1) is unstable since we have $\operatorname{spec}(A)=\{0.5 \pm 1 i\}$.

Let us express the set $\mathcal{K}$ as in (3) by choosing

$a_{1}=4-K^{2}$

where $K \in \mathbb{R}$. The first column of the table $T$ has 3 entries, namely, $f_{1}(k), f_{2}(k), f_{3}(k)$, which are shown in Figure 2. Next, we solve the SDP (36) finding the upper bound of $r$ given by

$\hat{\theta}=3.558$.

(the computation time is $0.61 \mathrm{~s}$ ). Lastly, by testing the feasibility of (38), we get the solution

$K^{*}=-1.099$.

As shown in Figure 2, the found controller $K^{*}$ is the maximizer of $f_{3}(k)$ in the set $K \in \mathcal{K} \cap \hat{\mathcal{K}}$.

Thus, it can be concluded that the controller $K^{*}$ can 
stabilize the closed-loop system (10) in the mean square sense. In fact, we have

$\max \mathcal{R}\left(\operatorname{spec}\left(\Phi\left(K^{*}\right)\right)\right)=-0.211<0$.

\section{Conclusion}

This paper has considered the design of stabilizing output feedback controllers for continuous-tme LTI systems over fading channels. It has been shown that a sufficient and necessary condition for the existence of such controllers can be obtained by solving a convex optimization problem in the form of an SDP.

\section{References}

(1) P. Antsaklis and J. Baillieul, "Special issue on technology of networked control systems," Proceedings of the IEEE, vol. 95, no. 1, pp. 5-8,

(2) R. Gupta and M.-Y. Chow, "Networked control system: Overview and research trends," IEEE Transactions on Industrial Electronics, vol. 57, no. 7, pp. 2527-2535, July 2010.

(3) L. Zhang, H. Gao, and O. Kaynak, "Network-induced constraints in networked control systems: a survey," IEEE Transactions on Industrial Informatics, vol. 9, no. 1, pp. 403-416, 2013.

(4) W. S. Wong and R. W. Brockett, "Systems with finite communication bandwidth constraints. II. stabilization with limited information feedback," IEEE Transactions on Automatic Control, vol. 44, no. 5, pp. 1049-1053, 1999.

(5) N. Elia and S. K. Mitter, "Stabilization of linear systems with limited information," IEEE Transactions on Automatic Control, vol. 46, no. 9,pp. 1384-1400, 2001.

(6) H. Gao, T. Chen, and J. Lam, "A new delay system approach to network-based control," Automatica, vol. 44, no. 1, pp. 39-52, 2008.

(7) Q. Li, G. Yi, C. Wang, L. Wu, and C. Ma, "LMI-based stability analysis of networked control systems with large time-varying delays," in International Conference on Mechatronics and Automation, pp. 713-717, 2006.

(8) D. E. Quevedo and D. Nesic, "Input-to-state stability of packetized predictive control over unreliable networks affected by packet-dropouts," IEEE Transactions on
Automatic Control, vol. 56, no. 2, pp. 370-375, 2011.

(9) N. Xiao, L. Xie, and L. Qiu, "Feedback stabilization of discrete-time networked systems over fading channels," IEEE Transactions on Automatic Control, vol. 57, no. 9, pp. 2176-2189, 2012.

(10) L. Qiu, G. Gu, and W. Chen, "Stabilization of networked multi-input systems with channel resource allocation," IEEE Transactions on Automatic Control, vol. 58, no. 3, pp. 554-568, 2013.

(11) M. Barforooshan and R. M. Esfanjani, "Output feedback stabilization over finite-bandwidth communication channels with signal-to-noise ratio constraint," IMA Journal of Mathematical Control and Information,p. dnv009, 2015.

(12) N. Elia, "Remote stabilization over fading channels," Systems \& Control Letters, vol. 54, no. 3, pp. 237-249, 2005.

(13) E. Routh, "A treatise on the stability of a given state of motion: particularly steady motion." Macmillan and Company, 1877.

(14) G. Chesi, "Robust static output feedback controllers via robust stabilizability functions," IEEE Transactions on Automatic Control, vol. 59, no. 6, pp. 1618-1623, 2014.

(15) G. Chesi, "LMI techniques for optimization over polynomials in control: a survey," IEEE Transactions on Automatic Control, vol. 55, no. 11, pp. 2500-2510, 2010.

(16) M. Putinar, "Positive polynomials on compact semialgebraic sets," Indiana University Mathematics Journal, vol. 42, no. 3, pp. 969-984, 1993.

(17) G. Chesi, A. Garulli, A. Tesi, and A. Vicino, "Characterizing the solution set of polynomial systems in terms of homogeneous forms: an LMI approach," International Journal of Robust and Nonlinear Control, vol. 13, no. 13, pp. 1239-1257, 2003.

(18) J. F. Sturm, "Using SeDuMi 1.02, a MATLAB toolbox for optimization over symmetric cones," Optimization methods and software, vol. 11, no. 1-4, pp. 625-653, 1999.

(19) S. Prajna, A. Papachristodoulou, and P. A. Parrilo, "Introducing SOSTOOLS: A general purpose sum of squares programming solver, in Proceedings of the 41st IEEE Conference on IEEE Conference on Decision and Control, vol. 1, 2002, pp. 741-746. 